\title{
Sobreuso de la tomografía computarizada en el traumatismo craneoencefálico infantil
}

\author{
Jorge M. Alcántara-Serrano ${ }^{1}$, Reginaldo A. Alcántara-Peraza ${ }^{2}$, Iván Romero-Solís ${ }^{2}$ y \\ Roberto P. González-Cobos ${ }^{3}$ \\ ${ }^{1}$ Facultad Mexicana de Medicina, Universidad La Salle; ${ }^{2}$ Departamento de Imagenología; ${ }^{3}$ Gerencia de Enseñanza e Investigación, Sanatorio \\ Durango. Ciudad de México, México
}

\begin{abstract}
Resumen
Introducción: La tomografía computarizada (TC) es el estudio de neuroimagen de elección para valorar lesiones intracraneales en el traumatismo, sin embargo, en la actualidad existe un uso indiscriminado de este recurso; se emplea para evaluar traumatismos leves, sin tomar en cuenta las dosis de radiación en niños. Objetivo: Evaluar si existe un sobreuso de la TC en los niños con traumatismo craneoencefálico basándonos en los criterios establecidos por la guía de práctica clínica «Atención inicial del traumatismo craneoencefálico en niños menores de 18 años». Material y métodos: Se realizó un estudio transversal analítico, mediante un muestreo opinático se revisaron 121 estudios de tomografía de cráneo simple de pacientes con edades entre 30 días y 18 años enviados al Servicio de Imagenología del Sanatorio Durango, de las cuales se obtuvieron las indicaciones y se evaluó si existió apego a los criterios de práctica clínica comparándolo con los hallazgos por imagen, aplicando la prueba estadística chi cuadrada para dos variables y k muestras. Resultados: Se observó que las imágenes de tomografía con hallazgos dentro de la normalidad es lo más frecuente, y al comparar las indicaciones que sí se apegaron a la guía y las que no se apegaron frente a los hallazgos por imagen se determinó que existe significancia estadística $(p<0.001)$. Discusión: Se observó un sobreuso de la TC de cráneo y un escaso apego a los criterios de práctica clínica. No es justificable la realización rutinaria de TC en traumatismos craneoencefálicos leves, tomando en cuenta los eventuales efectos nocivos de la radiación ionizante en niños.
\end{abstract}

Palabras clave: Sobreuso. Tomografía de cráneo. Trauma craneal. Niños. PECARN.

\section{Computed tomography overuse in childhood cranioencephalic trauma}

\begin{abstract}
Introduction: Computed tomography is the neuroimaging study of choice for assessing intracranial injuries in trauma, however, there is currently an indiscriminate use of this resource to evaluate mild trauma, regardless of the radiation dose in children. Objective: To evaluate if there is an overuse of computed tomography in children with traumatic brain injury based on the criteria established by the clinical practice guide "Initial attention to traumatic brain injury in children under 18 years of age". Material and Methods: An analytical cross-sectional study was carried out. Twenty-one studies of simple skull
\end{abstract}

\section{Correspondencia:}

Jorge Manuel Alcántara-Serrano

Durango, 290

Col. Roma Norte, Del. Cuauhtémoc Fecha de recepción: 01-06-2013

C.P. 60700, Ciudad de México Fecha de aceptación: 02-11-2018

E-mail: jalcantara345@gmail.com

DOI: 10.24875/RMN.M19000020

Disponible en internet: 20-03-2019

Rev Mex Neuroci. 2019;20(1):50-55

www.revmexneurociencia.com

1665-5044/@ 2019. Academia Mexicana de Neurología A.C. Publicado por Permanyer México. Este es un artículo Open Access bajo la licencia CC BY-NC-ND (http://creativecommons.org/licenses/by-nc-nd/4.0/). 
tomography of patients aged between 30 days and 18 years were reviewed by the Imaging Service of the Durango Sanatorium, from which the indications were obtained and evaluated if there was adherence to the clinical practice criteria comparing it with the imaging findings, applying the chi-square statistical test for two and $k$ samples. Results: It was observed that the images of tomography with findings within normality are the most frequent, and when comparing the indications adherence (whether they adhered to the guide or not) against the findings by image it was determined that there was statistical significance $(p<0.001)$. Discussion: An overuse of computed tomography of the skull and a low adherence to the criteria of clinical practice was observed. The routine performance of computed tomography in mild traumatic head injuries is not justifiable, considering the possible harmful effects of ionizing radiation in children.

Key words: Overuse. Skull tomography. Cranial trauma. Children. PECARN.

\section{Introducción}

El traumatismo craneoencefálico se define como la lesión directa de estructuras craneales, encefálicas o meníngeas, que se presenta como consecuencia de un efecto mecánico, provocado por un agente físico externo, que puede originar un deterioro funcional del contenido craneal ${ }^{1}$.

En el mundo existen cerca de 1.2 millones de personas que fallecen anualmente por traumatismo craneoencefálico y cerca de 20 a 50 millones sufren traumatismos craneoencefálicos no mortales. En México constituye la tercera causa de muerte, con un índice de mortalidad de 38.8 por cada 100 mil habitantes y con mayor incidencia en hombres de 15 a 45 años $^{2,3}$.

En la población pediátrica es la primera causa de morbimortalidad en la Unión Americana. Ocurren 150,000 traumas de cráneo severo en este grupo etario, con un total de 7,000 muertes y con un porcentaje más alto de discapacidad ${ }^{3,4}$.

La tomografía computarizada (TC) constituye la técnica de neuroimagen de referencia en el traumatismo craneoencefálico para permitir identificar lesiones intracraneales ${ }^{4-6}$. Sin embargo, debido a las altas dosis de radiación que se emite en los estudios de tomografía es de vital importancia que la solicitud de TC esté plenamente justificada y que se apliquen técnicas en las que se minimiza la dosis?.

Se estima que alrededor del $7 \%$ de los niños con traumatismo craneoencefálico leve presentarán una lesión visible en la TC y en menos del $1 \%$ existirá una lesión intracraneal que requiera intervención neuroquirúrgica urgente $6,8,9$.

La frecuencia de realización de TC en niños se ha duplicado en los últimos años. Se estima que en EE.UU. se realizaron, durante el año 2000, más de 62 millones de tomografías, de los cuales 4 millones correspondieron a pacientes pediátricos ${ }^{3,4}$. La Administración de Alimentos y Medicamentos (FDA por sus siglas en inglés) indica que el riesgo de desarrollar cáncer después de una TC con una dosis efectiva de 2.3 mSv podría ser de hasta 1 en 200, equivalente a exponerse a un año de radiación de fondo natural constituida por la radiación ambiental, radiación cósmica, la terrestre y la que nuestro cuerpo emite. Del total de radiación a la cual el hombre está sometido, el $11 \%$ equivale a la producida para fines médicos, por lo que es necesario que todo el personal al cuidado de la salud conozca los efectos de la radiación ionizante y las dosis a las que son expuestos los pacientes, principalmente los pediátricos ${ }^{7,10,11}$.

La OMS ${ }^{19,20}$ ha estimado el riesgo de presentar cáncer según las dosis absorbidas de radiación mediante estudios diagnósticos de imagen, según el sexo y la edad durante la exposición a dosis de $10 \mathrm{mSv}$, en población estadounidense, mencionando que el riesgo es mayor en mujeres que en hombres; asimismo, la probabilidad de cáncer en niños será de 1 niño de cada 500.

La OMS señala algunos aspecto importantes a la hora de realizar pruebas de imagen en niños:

- Los niños son más vulnerables al cáncer radioinducido, dos o tres veces más que los adultos.

- El cáncer relacionado con la exposición radiológica en la infancia resulta en años de vida perdidos durante la edad adulta.

- El cáncer radioinducido presenta un periodo de latencia que varía según el tipo de cáncer y la dosis recibidas.

- Es necesario modificar o configurar los ajustes en los parámetros de obtención de imágenes diagnosticas en niños pequeños y bebés.

- El daño celular que ocasiona la radiación ionizante es debido a la formación de radicales hidroxilo como resultado de la interacción de los rayos $X$ con moléculas de agua. Esos radicales libres a su vez interactúan con las cadenas de ADN provocando roturas, los rayos $X$ también pueden interactuar con el ADN y provocar mayor daño, sin embargo existen sistemas de reparación dentro de la célula, pero cuando se rompe la doble cadena de ADN se vuelve difícil de reparar, provocando mutaciones puntuales, 
Tabla 1. Criterios de decisión clínica PECARN para el uso de tomografía en pacientes menores de 18 años con trauma craneoencefálico

Niños menores de 2 años

Riesgo alto

- GCS: 14

- Otros signos de alteración del estado de consciencia*

- Fractura de cráneo palpable

Riesgo medio

- Hematoma del cuero cabelludo no frontal

- Historia de pérdida de la consciencia de 5 s o más

- Mecanismo severo de la lesión ${ }^{\dagger}$

- No actúa normal según los padres

Riesgo bajo

\section{Niños mayores de 2 años}

Riesgo alto

- GCS: 14

- Otros signos de alteración del estado de consciencia*

- Signos de fractura de base de cráneo

Riesgo medio

- Historia de pérdida de la consciencia

- Historia de vómitos

- Mecanismo severo de la lesión ${ }^{\dagger}$

- Cefalea severa

Riesgo bajo

*Agitación, somnolencia, estado confusional, bradipsíquico.

${ }^{\dagger}$ Accidente de tránsito en vehículo con eyección del paciente, muerte de pasajero, peatón y usuario de bicicleta sin casco arrollado por vehículo, caída de más $0.9 \mathrm{~m}$ si es menor de 2 años, de $1.5 \mathrm{~m}$ si es mayor de 2 años.

GCS: Glasgow Coma Scale; PECARN: Pediatric Emergency Care Applied Research Network.

Tabla 2. Criterios para realizar tomografía de cráneo según la guía de práctica clínica

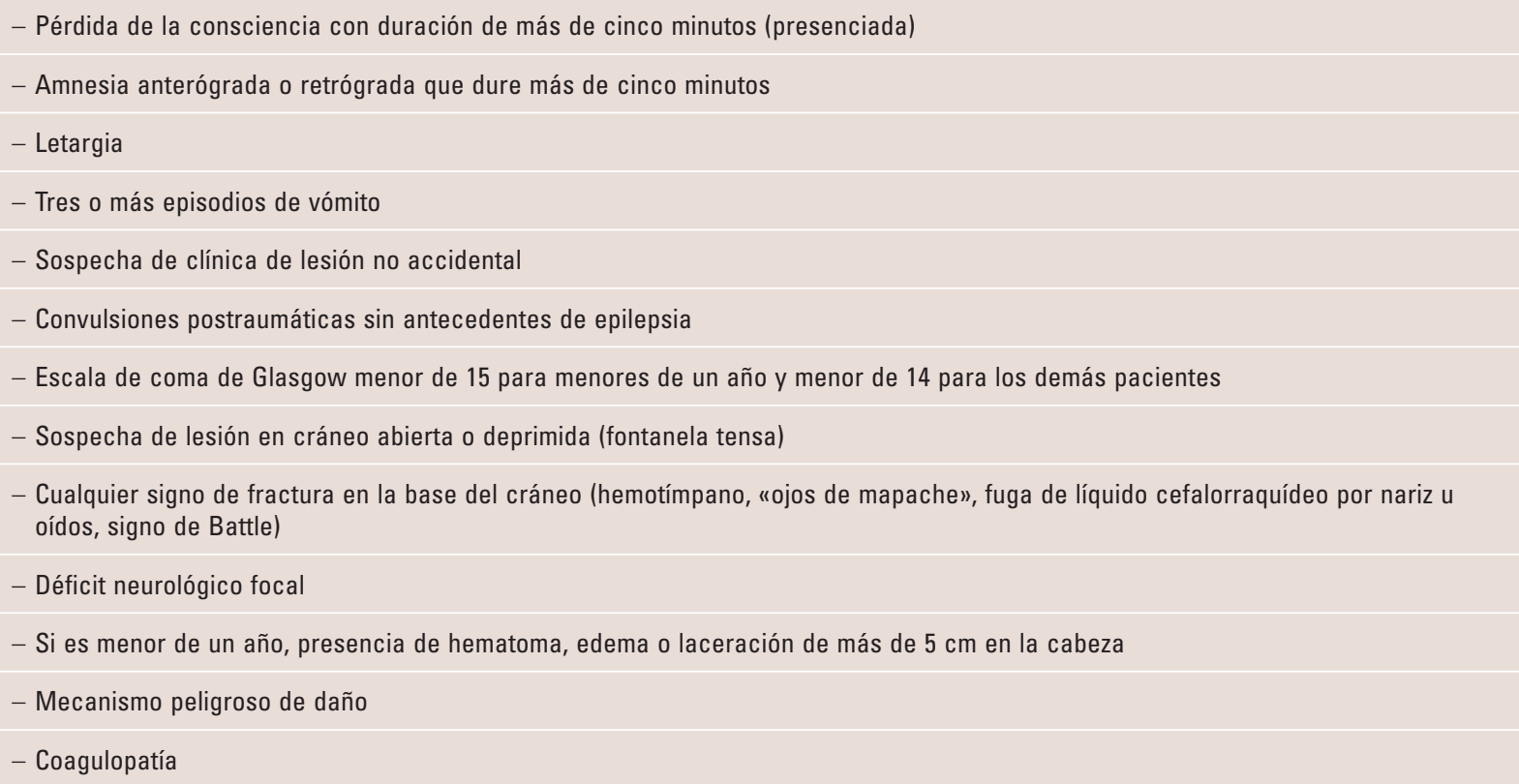

translocaciones cromosómicas y fusión de genes. Todo esto se vincula con la inducción de cáncer ${ }^{12}$.

- Por esta razón existen diversos grupos de investigación que se dedican a planear y elaborar guías con los criterios para la evaluación del paciente con traumatismo craneoencefálico y estandarizar el manejo $8,11,13,14$.

- Tal es el caso de la Pediatric Emergency Care Applied Research Network (PECARN) en EE.UU. ${ }^{18}$, que ha creado criterios (Tabla 1) y un algoritmo para la indicación de tomografía craneal en el traumatismo craneal infantil, los cuales son semejantes a los criterios de nuestra guía de práctica clínica.
- El objetivo del presente trabajo fue indagar si existe un sobreuso de la TC, así como una adecuada solicitud con base en los criterios establecidos, en pacientes pediátricos; y si el apego a los criterios está relacionado con los hallazgos por imagen obtenidos en dichos estudios.

\section{Material y métodos}

Se realizó un estudio transversal y analítico. En el periodo comprendido entre julio de 2014 a julio de 2016 se revisaron 121 tomografías de cráneo simples, 
accediendo a las historias clínicas de pacientes pediátricos en edades entre 30 días y 18 años que fueron enviados directos desde el Servicio de Urgencias Pediátricas y solicitadas por los médicos pediatras adscritos a los diferentes turnos, obteniendo las principales indicaciones para realización de tomografía en el Servicio de Imagen de un hospital privado, sin haber obtenido información clínica suficiente (puntuación en la escala de coma de Glasgow).

Las imágenes se obtuvieron mediante un tomógrafo Philips Ingenuity 64 core $^{\circledR}$, con reconstrucciones multiplanares y volumétricas, con parámetros KV 120 mAs 300 y pitch 0.298 . Los estudios fueron evaluados por médicos radiólogos certificados.

\section{Resultados}

Las indicaciones más frecuentes en las solicitudes de TC fueron: solo el traumatismo craneoencefálico, 79 (65.2\%); traumatismo con mecanismo de alta energía, como desaceleración en vehículo de motor y caída de más de $100 \mathrm{~cm}, 10$ (8.2\%); crisis convulsivas postraumáticas, $3(2.4 \%)$; herida menor de $5 \mathrm{~cm}$, $3(2.4 \%)$; pérdida del estado de alerta (no presenciado), 10 (8.2\%); vómito en más de 3 ocasiones, 12 (9.9\%), y sospecha de fractura (observada por radiografía de cráneo), 4 (3.3\%) (Fig. 1). Los hallazgos en las imágenes por tomografía fueron: normal 88 (72.7\%); hematoma subgaleal $23(19 \%)$; fractura $7(5.7 \%)$ y hematoma epidural 3 (2.4\%) (Fig. 2).

En la tabla 2 se compara al grupo de pacientes a los que se les solicitó TC siguiendo adecuadamente los criterios establecidos para ello (grupo 1), frente al grupo de los que se les solicitó tal estudio sin apego a estos (grupo 2). Se observa que la mayoría de los estudios realizados a los pacientes del grupo 1 tuvieron un resultado negativo, al igual que en el grupo 2. Sin embargo, el cuadro muestra una diferencia significativa $(p<0.001)$ a favor del grupo 1, en cuanto a un mayor número de resultados positivos, es decir, con algún tipo de patología.

En la tabla 3 los sujetos de estudio se agruparon de acuerdo con las etapas pediátricas. Se observa un mayor número de solicitudes sin apego a los criterios, sin presentar significancia estadística.

En la tabla 4 se comparan los grupos de edades pediátricas con los hallazgos obtenidos por imágenes de tomografía, observando que existe un mayor número de pacientes con estudios donde los hallazgos fueron normales, pero sin mostrar significancia estadística.

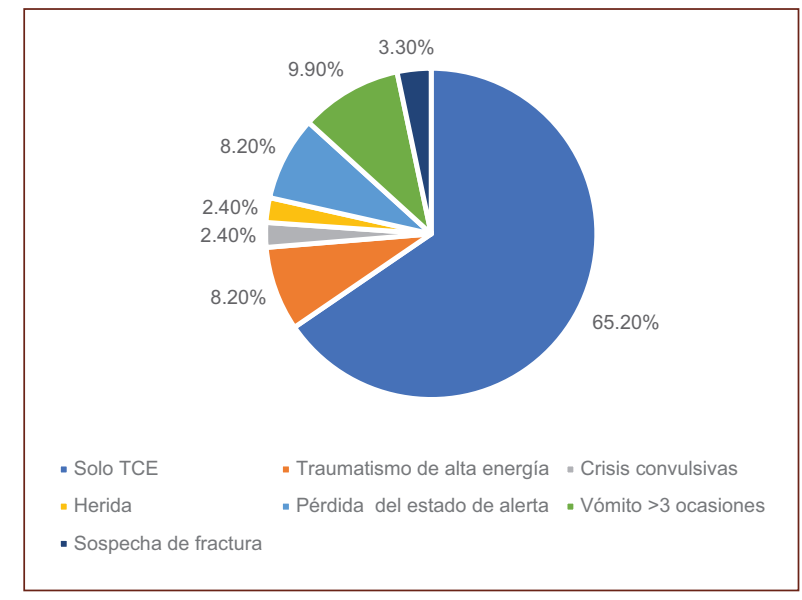

Figura 1. Causas más frecuentes de solicitud de tomografía computarizada en niños.

TCE: Traumatismo craneoencefálico

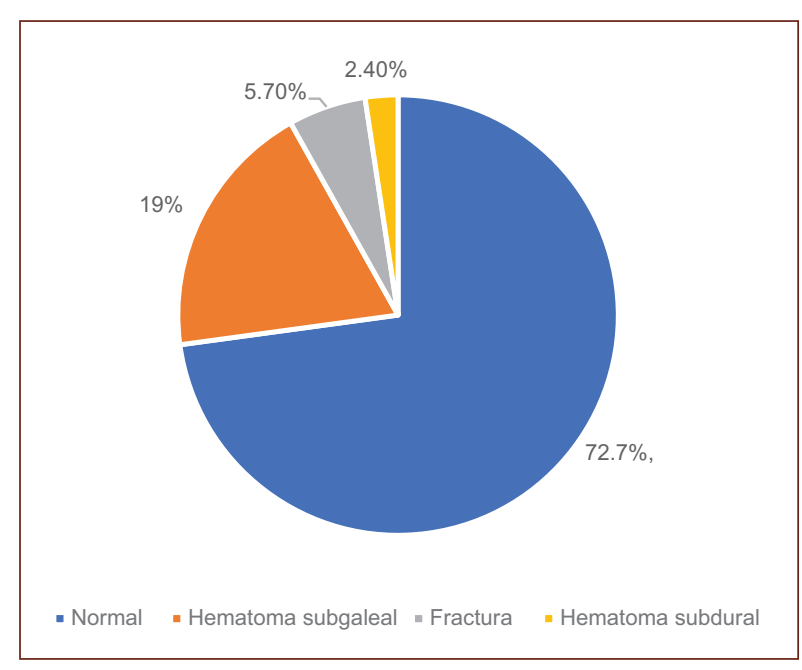

Figura 2. Hallazgos tomográficos. Se observa que la normalidad es lo más frecuente en los estudios solicitados.

\section{Discusión}

La radiología se ha convertido en parte habitual de la práctica médica como pruebas complementarias al diagnóstico clínico; sin embargo, incluso las dosis pequeñas de radiación no están exentas de riesgos.

En nuestro estudio se observó un sobreuso de la TC y un bajo apego a los criterios de práctica clínica en pacientes pediátricos con traumatismo craneoencefálico.

De ahí la importancia y obligación del médico tratante de realizar una adecuada valoración clínica y clasificar el traumatismo craneoencefálico según la escala de coma de Glasgow y de esta forma otorgar la 
Tabla 3. Resultados de la comparación entre los resultados de la tomografía computarizada (TC) en relación al apego a los criterios de solicitud de TC $(p<0.001$, prueba de chi cuadrada para dos muestras independientes)

\begin{tabular}{|l|c|c|c|}
\hline \multirow{2}{*}{ Criterios } & \multicolumn{2}{|c|}{ TC } & \multirow{2}{*}{ Total } \\
\cline { 2 - 3 } & + & - & \\
\hline Sí & 8 & 21 & 29 \\
\hline No & 2 & 90 & 92 \\
\hline Total & 10 & 111 & 121 \\
\hline
\end{tabular}

Tabla 4. Comparación entre los intervalos de edad y el apego a los criterios de solicitud de tomografía computarizada ( $p$ no significativa, prueba de chi cuadrada para k muestras)

\begin{tabular}{|l|c|c|c|}
\hline \multirow{2}{*}{} & \multicolumn{2}{|c|}{ Criterios } & \multirow{2}{*}{ Total } \\
\cline { 2 - 3 } & Sí & No & \\
\hline Lactantes & 3 & 17 & 20 \\
\hline Preescolar & 7 & 22 & 29 \\
\hline Escolar & 8 & 21 & 29 \\
\hline Adolescentes & 8 & 35 & 43 \\
\hline Total & 26 & 95 & 121 \\
\hline
\end{tabular}

Tabla 5. Comparación entre los intervalos de edad y los resultados tomográficos ( $p$ no significativa)

\begin{tabular}{|l|c|c|c|}
\hline \multirow{2}{*}{} & \multicolumn{2}{|c|}{ Hallazgos } & \multirow{2}{*}{ Total } \\
\cline { 2 - 3 } & + & - & \\
\hline Lactantes & 4 & 16 & 20 \\
\hline Preescolar & 4 & 25 & 29 \\
\hline Escolar & 1 & 28 & 29 \\
\hline Adolescentes & 2 & 41 & 43 \\
\hline Total & 11 & 110 & 121 \\
\hline
\end{tabular}

información clínica necesaria al médico radiólogo, quien orientará sobre la decisión de realizar el estudio de imagen.

La realización de TC en pacientes con traumatismo craneoencefálico no severo de forma rutinaria no es justificable, en caso de ser necesario hay que pedir la opinión del médico radiólogo.

La misma FDA ha emitido recomendaciones para disminuir la radiación de la exposición y el riesgo subsiguiente de cáncer: reducción de la corriente del tubo, disminución del voltaje del tubo, incrementar el pitch (tiempo de rotación del tubo) y el control automático de la exposición, entre otras?

En el marco normativo de México y otros países se especifica que el médico solicitante deberá justificar y en su caso consultar con el médico radiólogo si el estudio seleccionado proporcionará la información suficiente para beneficio del paciente, y a su vez proporcionar información clínica suficiente para que el médico radiólogo aplique la técnica o procedimiento a la necesidad del diagnóstico médico ${ }^{1,11,15-17}$. En nuestro país, el Centro Nacional de Tecnología en Salud (por medio de su Catálogo Maestro de Guías de Práctica Clínica ${ }^{1}$ ) estipula las indicaciones para la realización de tomografía craneal de forma inmediata (Tabla 5).

\section{Conclusión}

Es necesaria la concienciación sobre los estudios de imagen con radiación ionizante, principalmente en niños, puesto que existe un porcentaje significativo de desarrollo de neoplasias asociadas a radiación artificial, por lo que el personal de salud debe conocer los criterios y protocolos estipulados en las guías de práctica clínica y apegarse a la normatividad establecida.

\section{Declaración de conflictos de interés}

Los autores declaran que no existen conflictos de interés relevantes para este artículo de revisión.

\section{Fuentes de financiamiento}

No existió una fuente de financiamiento particular para este informe científico.

\section{Bibliografía}

1. Atención inicial del traumatismo craneoencefálico en pacientes menores de 18 años. Guía de referencia rápida [Internet]. México: Consejo de Salubridad General [2008]. Disponible en: http://www.cenetec.salud.gob.mx/contenidos/gpc/catalogoMaestroGPC.html.

2. Ontiveros A, Preciado AK, Matute E, López-Cruz M, López-Elizalde R. Factores pronósticos de recuperación y reinserción laboral en adultos con traumatismo craneoencefálico. Rev Mex Neuroci. 2014;15:211-7.

3. Motta-Ramírez GA, Jiménez-Parra JL, Limas-Santos NI, Álvarez-Flores NH, Solis-Vargas MC. El uso y abuso de la tomografía computarizada de cráneo en la consulta externa pediátrica neurológica. Rev Mex Neuroci. 2011;12:358-64.

4. Bejarano Mondragón L, Ramírez D, Ramírez MM. Traumatismo craneoencefálico en niños: Relación entre los hallazgos tomográficos y el pronóstico. Rev Esp Med-Quirurg. 2008;13:60-8.

5. Quayle KS, Jaffe DM, Kuppermann N, Kaufman BA, Lee BC, Park TS, et al. Diagnostic testing for acute head injury in children: When are head 
computed tomography and skull radiographs indicated? Pediatrics. 1997;99:1-8.

6. Atabaki SM, Stiell IG, Bazaman JJ. Tomografía computarizada en el traumatismo craneoencefálico menor. Arch Pedia Adolesc Med. 2008;162(5):439-45.

7. Mendizabal Méndez AL. Radiación ionizante en tomografía computarizada: un tema de reflexión. An Rad Mex. 2012;2:90-7.

8. Velasco R, Arribas M, Valencia C, Zamora N, Fernández SM, Lobeiras A et al. Adecuación del manejo diagnóstico del traumatismo craneoencefálico leve en menor de 24 meses a las guías de práctica clínica de PECARN y AEP. An Pediatr. 2015;83:166-72.

9. De la Torre-Gómez RE, Rodríguez-Rodríguez IC, López-León A, Carranza-Barrera LG, Brancaccio-Orozco J, Guzmán-Rodríguez I, et al. Revisión de trauma de cráneo severo en niños. Rev Med. 2014;5:229-37.

10. Brenner DJ, Hail EJ. Computed tomography-An increasing source of radiation exposure. $\mathrm{N}$ Engl J Med. 2007;357:2277-84.

11. Guía de indicaciones para la correcta solicitud de pruebas de diagnóstico por imagen. Protección radiológica 118 [Internet]. Comisión Europea. Dirección General de Medio Ambiente; 2000 [fecha de publicación 2001]. Disponible en: http://pendientedemigracion.ucm.es/info/fismed/ pr118.pdf.

12. Brody As, Frush DP, Walter Huda, Brent RL; American Academy of Pediatrics Section on Radiology. Radiation risk to children from computed tomography. Pediatrics. 120;3:677-82.

13. Manrique Martínez I, Alcalá Minagorre PJ. Manejo del traumatismo craneal pediátrico [Internet]. Protocolos diagnóstico-terapéuticos de Urgencias Pediátricas SEUP-AEP [2010]. Disponible en: https://www.ae- ped.es/sites/default/files/documentos/manejo_del_traumatismo_craneal_ pediatrico.pdf

14. Lee LP, Rojas de Recalde L, Espinola de Canata M, Sostoa G. Tomografía axial computarizada en traumatismos craneoencefálicos leves. Pediatr. 2007;34:122-5.

15. Guías de Práctica Clínica para la solicitud de estudios de diagnóstico por imagen en Pediatría. BSCP Can Ped. 2002;26:2-3.

16. Franco-Koehrlen CA, Iglesias-Leborfino J, Bernaraez-Zapata y Macías-Rendón ME. Decisión clínica para la realización de tomografía axial computarizada de cráneo en niños con traumatismo craneoencefálico no severo. Bol Med del Hosp Inf Mex. 2015:72:169-73.

17. NOM-229-SSA1-2002, Salud ambiental. Requisitos técnicos para las instalaciones, responsabilidades sanitarias, especificaciones técnicas para los equipos y protección radiológica en establecimientos de diagnóstico médico con rayos $X$ [Internet]. México: Diario Oficial de la Federación. Disponible en: http://dof.gob.mx/normasOficiales.php?co$\mathrm{dp}=4585$ \& view $=$ si\#. .

18. Carlos Mójica, Gañan-Vesga JG, Arenas Correa HC. Utilidad de las reglas de decisión clínica PECARN como predictor de lesión intracraneana en el trauma cráneo encefálico catalogado como leve en la población pediátrica de Tunja, Boyacá. Pediatr. 2016;49:78-83.

19. Brody AS, Frush DP, Huda W and Brent RL. Radiation risk to children from computed tomography. Pediatrics. 2007;120(3):677-82.

20. Comunicando los riesgos de la radiación en radiodiagnóstico pediátrico [Internet]. Ginebra: Organización Mundial de la Salud; 2016. Disponible en: http://www.who.int/maternal_child_adolescent/documents/radiation-risks-paediatric-imaging/es. 